\title{
ON OPTIMAL HIGH-ORDER IN TIME APPROXIMATIONS FOR THE KORTEWEG-DE VRIES EQUATION
}

\author{
OHANNES KARAKASHIAN AND WILLIAM MCKINNEY
}

\begin{abstract}
We show that the well-known order reduction phenomenon affecting implicit Runge-Kutta methods does not occur when approximating periodic solutions of the Korteweg-de Vries equation.
\end{abstract}

\section{INTRODUCTION}

In this paper we obtain optimal rate of convergence estimates in time for highorder fully discrete approximations to 1-periodic solutions of the Korteweg-de Vries $(\mathrm{KdV})$ equation

$$
\left\{\begin{aligned}
u_{t}+u u_{x}+\varepsilon u_{x x x} & =0, \quad 0<x<1, t>0, \\
u(x, 0) & =u^{0}(x) .
\end{aligned}\right.
$$

These approximations are generated by a finite element process for the spatial discretization and implicit Runge-Kutta (IRK) methods for the time-stepping. As a class, IRK methods possess superior stability properties, enabling them to handle many stiff nonlinear problems. In addition, many IRK methods are such that at each step the computational work may be subdivided into several independent tasks, allowing for parallel implementation on multiprocessor computers; cf. [10,11].

It is well known, however, that for some problems the observed order of convergence of an IRK method is less than the optimal order. We remark here that this phenomenon is not caused per se by a lack of regularity on the part of the solution. A first allusion to this phenomenon may be traced to Crouzeix' thesis [6], where lower than the optimal orders are obtained for parabolic problems with time-dependent coefficients; for numerical evidence we refer to [7] and the references therein.

In the case of the $\mathrm{KdV}$, in a previous paper [8], the order reduction was avoided by the introduction of appropriate correction terms during the calculation of the intermediate values, in the case of two diagonally implicit methods

\footnotetext{
Received February 23, 1989.

1980 Mathematics Subject Classification (1985 Revision). Primary 65N30; Secondary 35Q20, $65 \mathrm{M} 15$.

The first author's research was supported by the Air Force Office for Scientific Research under grant AFOSR-88-0019.
} 
of (optimal) orders 3 and 4 . In this paper, we show that these modifications are indeed unnecessary, and that the optimal orders can be recovered by exploiting the fact that the solution is in the domain of adequately high powers of the differential operator. This agrees well with the results of numerical experiments. Our new approach consists of introducing, at every time level $t^{n}$, the function $u^{n+1}$ by applying the IRK method directly to (1.1). We observe, however, that $u^{n+1}$ is not actually computed but serves merely as a convenient intermediary for the analysis. This generates two difficulties: First, we must establish that the system of nonlinear (ordinary) differential equations obtained does indeed have a solution which is sufficiently regular. Second, the fully discrete (computable) approximations $u_{h}^{n+1}$ must be compared directly to $u^{n+1}$, a difficult task in attempting energy type estimates, given that the difference of the two functions is not in the finite-dimensional subspace.

The paper is organized as follows: $\S 2$ is devoted to preliminary material. In $\S 3$, we successfully resolve the first difficulty mentioned above. We also obtain bounds for $u^{n+1}$ in terms of the initial data $u^{0}$. In $\S 4$ we obtain optimal estimates for the local truncation error $u^{n+1}-u\left(t^{n+1}\right)$ in appropriate norms. Using the stability properties of the IRK methods, we then estimate $u^{n+1}$ $u_{h}^{n+1}$. The convergence of the fuliy discrete approximations is then obtained by a simple argument. We note that in this paper we do not address issues pertinent to the existence and uniqueness of the fully discrete approximations $\left\{u_{h}^{n}\right\}$ and to the convergence of Newton's method for solving the nonlinear algebraic equations, since these topics were treated in earlier works; cf. [2, 3, 8].

For the sake of simplicity, we drop the constant $\varepsilon$, given that all the results remain valid, with the proviso that the constants appearing in the estimates may tend to infinity as $\varepsilon$ approaches zero.

\section{Preliminaries}

2.1. Some function spaces. For integer $m \geq 0$ and real $p, 1 \leq p \leq \infty$, we let $W^{m, p}=W^{m, p}(0,1)$ denote the usual real Sobolev spaces with norm $\|\cdot\|_{m, p}$. For $p=2$, the Hilbert spaces $H^{m}=W^{m, 2}$ have inner product $(\cdot, \cdot)_{m}$ and norm $\|\cdot\|_{m}$. For $m=0$ we shall let $L^{2}=H^{0}$ and use $(\cdot, \cdot),\|\cdot\|$. We shall also replace $W^{0, \infty}$ and $\|\cdot\|_{0, \infty}$ by $L^{\infty}$ and $\|\cdot\|_{\infty}$, respectively.

We shall also use the spaces $C^{m}=C^{m}[0,1]$ of functions $f$ such that $f, \ldots, f^{(m)}$ are continuous on $[0,1]$, equipped with the norm $\|\cdot\|_{m, \infty}$.

We shall say that $f$ is periodic in $C^{m}$ if $f \in C^{m}$ and $f^{(j)}(0)=f^{(j)}(1), j=$ $0, \ldots, m$. Furthermore, in view of the (compact) imbedding $H^{m} \hookrightarrow C^{m-1}$, $m \geq 1$ (cf., e.g., [1]), we shall say that $f$ is periodic in $H^{m}, m \geq 1$, if $f \in H^{m}$ and $f^{(j)}(0)=f^{(j)}(1), j=0, \ldots, m-1$.

For $q \geq 1$ integer, we use $\mathscr{H}^{m}$ to denote the $q$-product $H^{m} \times \cdots \times H^{m}$ equipped with the inner product $((u, v))_{m}=\sum_{i=1}^{q}\left(u_{i}, v_{i}\right)_{m}$, and norm $\|u\|_{m}=$ $\left(\sum_{i=1}^{q}\left(u_{i}, u_{i}\right)_{m}\right)^{1 / 2}$. In particular, we shall denote $((\cdot, \cdot))_{0}$ by $((\cdot, \cdot))$ and $\|\cdot\|_{0}$ 
by $\|\cdot\|$. We shall also use the spaces $\mathscr{C}^{m}=C^{m} \times \cdots \times C^{m}$ equipped with the norm $\|u\|_{m, \infty}=\max _{1 \leq i \leq q}\left\|u_{i}\right\|_{m, \infty}$; we shall denote $\|\cdot\|_{0, \infty}$ by $\|\cdot\|_{\infty}$.

It was proved by Bona and Smith [4] that a unique global, periodic solution $u$ to (1.1) exists in $H^{m}, m \geq 2$, provided the intial data $u^{0}$ is periodic in $H^{m}$. Furthermore, $\partial^{j} u / \partial t^{j} \in C\left(0, t^{*} ; H^{m-3 j}\right)$ for $0<t^{*}<\infty$ and all $j \geq 0$ such that $m-3 j \geq 0$, and

$$
\sup _{0 \leq t \leq t^{*}}\left\|\frac{\partial^{j} u}{\partial t^{j}}\right\|_{m-3 j} \leq c\left(\left\|u^{0}\right\|_{m}\right) .
$$

We assume that $u^{0}$ is periodic in $H^{m}$ for $m$ sufficiently large to guarantee the convergence results below.

2.2. The finite-dimensional spaces. Let $4 \leq r \leq N$ be integers and let $h=1 / N$. Let $S_{h}^{r}$ denote the space of 1-periodic smooth splines of degree at most $r-1$ defined on a uniform partition $x_{i}=i h, i=0, \ldots, N$.

It is well known that if $v$ is 1-periodic and sufficiently smooth, then there exists a $\chi \in S_{h}^{r}$ such that

$$
\sum_{j=0}^{m-1} h^{j}\|v-\chi\|_{j, p} \leq c h^{m}\|v\|_{m, p}, \quad 1 \leq m \leq r, p=2 \text { or } p=\infty,
$$

for some constant $c$ independent of $h, v$, and $\chi$.

In addition, elements of $S_{h}^{r}$ possess the following inverse property: there exists a constant $c$, independent of $h$, such that for all $\chi \in S_{h}^{r}$

$$
\begin{aligned}
& \|\chi\|_{\beta} \leq c h^{-(\beta-\alpha)}\|\chi\|_{\alpha}, \quad\|\chi\|_{\alpha, \infty} \leq c h^{-(\alpha+1 / 2)}\|\chi\|, \\
& 0 \leq \alpha \leq \beta \leq r-1 .
\end{aligned}
$$

One may choose as basis the well-known "Bell" splines $\phi_{1}, \ldots, \phi_{N}$ associated with the nodes $x_{1}, \ldots, x_{N}$, respectively. For the analysis, we find it more convenient to use a set of modified basis functions $\tilde{\phi}_{1}, \ldots, \tilde{\phi}_{N}$ used by Thomée and Wendroff [12].

Let $\tilde{G}$ denoted the Gramian matrix $\widetilde{G}_{i j}=h^{-1}\left(\tilde{\phi}_{j}, \tilde{\phi}_{i}\right) . \tilde{G}$ is symmetric, positive definite; so $\widetilde{G}^{1 / 2}$ and $\widetilde{G}^{-1 / 2}$ may be defined in the usual way. Let $(\cdot, \cdot)_{h}$ denote the following inner product on $R^{N}$ :

$$
(V, W)_{h}=h \sum_{i=1}^{N} V_{i} W_{i},
$$

and $\|V\|_{h}$ the associated norm. We have (cf. [12])

$$
g_{1}\|V\|_{h}^{2} \leq(\tilde{G} V, V)_{h} \leq g_{2}\|V\|_{h}^{2} \quad \forall V \in R^{N},
$$

where $g_{1}, g_{2}$ are positive constants independent of $h$. We also have

$$
\left\|v_{h}\right\|^{2}=(\tilde{G} V, V)_{h}, \quad \text { where } v_{h}=\sum_{i=1}^{N} V_{i} \tilde{\phi}_{i} .
$$


Let $v$ be periodic and sufficiently smooth; then the quasi-interpolant $\tilde{v}_{h} \equiv$ $\sum_{i=1}^{N} v(i h) \tilde{\phi}_{i}$ of $v$ approximates $v$ in the following way:

$$
\left\|v-\tilde{v}_{h}\right\| \leq h^{r}\left\|v^{(r)}\right\| \text {. }
$$

Let $v \in H^{r} \cap W^{2, \infty}$ be periodic. Using (2.1), (2.2), and (2.5), it can be shown that

$$
\left\|\tilde{v}_{h x}\right\|_{\infty} \leq c\left(\|v\|_{2, \infty}+\|v\|_{r}\right)
$$

cf., e.g., [2, p. 424].

2.3. The implicit Runge-Kutta methods. For $q \geq 1$ integer, a $q$-stage IRK method is given as a set of constants arranged in tableau form:

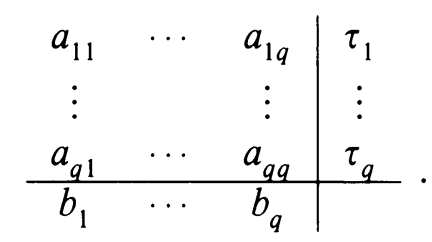

Given the Initial Value Problem

$$
\left\{\begin{aligned}
y^{\prime} & =f(t, y), \quad 0<t \leq t^{*}, \\
y(0) & =y^{0},
\end{aligned}\right.
$$

IRK methods can be applied to generate approximations $y^{0}, \ldots, y^{J}$ as follows: let $k=t^{*} / J$ be the temporal stepsize and let $t^{n}=n k$. Then

$$
y^{n+1}=y^{n}+k \sum_{i=1}^{q} b_{i} f\left(t^{n, i}, y^{n, i}\right)
$$

where $t^{n, i}=t^{n}+k \tau_{i}$, and where the intermediate values $y^{n, i}$ are given by the coupled system of equations

$$
y^{n, i}=y^{n}+k \sum_{j=1}^{q} a_{i j} f\left(t^{n, j}, y^{n, j}\right), \quad i=1, \ldots, q .
$$

We shall assume that these methods satisfy certain stability and consistency conditions. Indeed, it will be required that

(S) $\left\{\begin{array}{l}b_{i} \geq 0, \quad i=1, \ldots, q, \\ \text { the } q \times q \text { array } m_{i j}=a_{i j} b_{i}+a_{j i} b_{j}-b_{i} b_{j} \text { is nonnegative definite. }\end{array}\right.$

The above condition, known as algebraic stability, is stronger than that of $A$ stability. 
The consistency conditions are given by
(B) $\quad \sum_{j=1}^{q} b_{j} \tau_{j}^{l}=\frac{1}{l+1}, \quad l=0, \ldots, \nu-1$,
(C) $\sum_{j=1}^{q} a_{i j} \tau_{j}^{l}=\frac{\tau_{i}^{l+1}}{l+1}, \quad i=1, \ldots, q, l=0, \ldots, p-1$,
(D) $\sum_{i=1}^{q} a_{i j} \tau_{i}^{l} b_{i}=\frac{b_{j}}{l+1}\left(1-\tau_{j}^{l+1}\right), \quad j=1, \ldots, q, l=0, \ldots, r-1$,

for some integers $\nu, p, r \geq 1$. We assume that

$$
\begin{gathered}
\nu \leq r+p+1, \\
\nu \leq 2 p+2 .
\end{gathered}
$$

The consistency relations may be expressed, with the help of the $q$-vector $e=(1, \ldots, 1)^{T}$, as

$$
\begin{aligned}
b^{T} T^{l} e & =\frac{1}{l+1}, \quad l=0, \ldots, \nu-1, \\
A T^{l} e & =\frac{T^{l+1} e}{l+1}, \quad l=0, \ldots, p-1, \\
b^{T} T^{l} A & =\frac{b^{T}}{l+1}\left(I-T^{l+1}\right), \quad l=0, \ldots, r-1,
\end{aligned}
$$

respectively, where $A=\left(a_{i j}\right), T=\operatorname{diag}\left\{\tau_{1}, \ldots, \tau_{q}\right\}$, and $b=\left(b_{1}, \ldots, b_{q}\right)^{T}$.

The existence of the numerical approximations is obtained by assuming the following positivity property:

(P) $\left\{\begin{array}{l}A \text { is invertible and there exists a positive diagonal matrix } D \\ \text { such that } x^{T} C x>0 \forall x \in R^{q}, x \neq 0, \text { where } C=D A^{-1} D^{-1} .\end{array}\right.$

In particular, let the constants $\alpha_{1}, \alpha_{2}, \beta_{1}, \beta_{2}, \gamma_{1}, \gamma_{2}$ be given by

$$
\begin{aligned}
& 0<\alpha_{1} \leq x^{T} C x, \quad x^{T} C y \leq \alpha_{2}, \\
& 0<\beta_{1} \leq x^{T} C^{-1} x, \quad x^{T} C^{-1} y \leq \beta_{2}, \\
& 0<\gamma_{1} \leq x^{T} D x \leq \gamma_{2}, \quad \forall x, y \in R^{q} \text { with } x^{T} x=y^{T} y=1 .
\end{aligned}
$$

We next give the two- and three-stage methods of some families of IRK methods.

(i) Gauss-Legendre methods. These methods form a particularly interesting class in that the matrix $M$ in (S) vanishes identically, a fact that has important implications such as the existence of discrete conservation laws and mild growth of the discretization error. For this class, $\nu=2 q, p=r=q$. 
2-stage method

$$
\begin{array}{cc|c}
\frac{1}{4} & \frac{1}{4}-\frac{1}{2 \sqrt{3}} & \frac{1}{2}-\frac{1}{2 \sqrt{3}} \\
\frac{1}{4}+\frac{1}{2 \sqrt{3}} & \frac{1}{4} & \frac{1}{2}+\frac{1}{2 \sqrt{3}} \\
\hline \frac{1}{2} & \frac{1}{2} &
\end{array}
$$

3-stage method

\begin{tabular}{ccc|c}
$\frac{5}{36}$ & $\frac{80-24 \sqrt{15}}{360}$ & $\frac{50-12 \sqrt{15}}{360}$ & $\frac{1}{2}-\frac{\sqrt{15}}{10}$ \\
$\frac{50+15 \sqrt{15}}{360}$ & $\frac{2}{9}$ & $\frac{50-15 \sqrt{15}}{360}$ & $\frac{1}{2}$ \\
$\frac{50+12 \sqrt{15}}{360}$ & $\frac{80+24 \sqrt{15}}{360}$ & $\frac{5}{36}$ & $\frac{1}{2}+\frac{\sqrt{15}}{10}$ \\
\hline$\frac{5}{18}$ & $\frac{8}{18}$ & $\frac{5}{18}$ &
\end{tabular}

(ii) Radau IIA methods. These methods are characterized by $\tau_{q}=1$. Also, $\nu=2 q-1, p=q, r=q-1$.

2-stage method

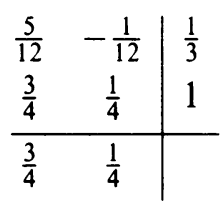

3-stage method

\begin{tabular}{ccc|c}
$\frac{88-7 \sqrt{6}}{360}$ & $\frac{296-169 \sqrt{6}}{1800}$ & $\frac{-2+3 \sqrt{6}}{225}$ & $\frac{4-\sqrt{6}}{10}$ \\
$\frac{296+169 \sqrt{6}}{1800}$ & $\frac{88+7 \sqrt{6}}{360}$ & $\frac{-2-3 \sqrt{6}}{225}$ & $\frac{4+\sqrt{6}}{10}$ \\
$\frac{16-\sqrt{6}}{36}$ & $\frac{16+\sqrt{6}}{36}$ & $\frac{1}{9}$ & 1 \\
\hline$\frac{16-\sqrt{6}}{36}$ & $\frac{16+\sqrt{6}}{36}$ & $\frac{1}{9}$ &
\end{tabular}

Both of the families above are infinite, in the sense that arbitrarily high-order methods can be constructed.

(iii) Diagonally implicit methods.

2-stage method

$$
\begin{array}{cc|c}
\frac{1}{2}+\frac{1}{2 \sqrt{3}} & 0 & \frac{1}{2}+\frac{1}{2 \sqrt{3}} \\
-\frac{1}{\sqrt{3}} & \frac{1}{2}+\frac{1}{2 \sqrt{3}} & \frac{1}{2}-\frac{1}{2 \sqrt{3}} \\
\hline \frac{1}{2} & \frac{1}{2} &
\end{array}
$$

3-stage method

\begin{tabular}{ccc|c}
$\gamma$ & 0 & 0 & $\gamma$ \\
$\frac{1}{2}-\gamma$ & $\gamma$ & 0 & $\frac{1}{2}$ \\
$2 \gamma$ & $1-4 \gamma$ & $\gamma$ & $1-\gamma$ \\
\hline$\frac{1}{24\left(\frac{1}{2}-\gamma\right)^{2}}$ & $1-\frac{1}{12\left(\frac{1}{2}-\gamma\right)^{2}}$ & $\frac{1}{24\left(\frac{1}{2}-\gamma\right)^{2}}$ &
\end{tabular}

where $\gamma=\frac{1}{2}+\frac{1}{\sqrt{3}} \cos \frac{\pi}{18}$ is a root of the equation $24 x^{3}-36 x^{2}+12 x-1=0$. For the two-stage method, $\nu=3, p=r=1$. For the three-stage method, $\nu=4, p=r=1$; hence $(2.10 \mathrm{a})$ is not satisfied. This will necessitate a slight modification in the estimation of the local truncation error. In [8] these two methods were applied to the $\mathrm{KdV}$ equation with appropriate modifications of the intermediate equations (2.9). Optimal-order rates of convergence were then established. The new machinery adopted in this paper reveals that these modifications are unnecessary.

Concerning the application of IRK methods to the. Initial Value Problem (2.7), a comment concerning the local truncation error

$$
\varepsilon^{n+1}=k^{-1}\left(y^{n+1}-y\left(t^{n+1}\right)\right)
$$


is in order. $y^{n+1}$ is defined by taking $y^{n}=y\left(t^{n}\right)$ in (2.8) and (2.9). Suppose that the IRK method satisfies (B), (C), and (D) together with (2.10a) and $(2.10 \mathrm{~b})$. Suppose also that $f$ is sufficiently smooth and that

$$
\left|f\left(t, y_{1}\right)-f\left(t, y_{2}\right)\right| \leq L\left|y_{1}-y_{2}\right| \quad \forall t \in\left[0, t^{*}\right], \forall y_{1}, y_{2} .
$$

Then (cf. $[5,6])$ there exists $k_{0}>0$ such that $\left|\varepsilon^{n+1}\right| \leq c k^{\nu}$ for $k<k_{0}$, where $c$ is a constant independent of $k$, but which may depend, in general exponentially, on $L$.

When (2.11) does not hold, or when $L$ is large, such bounds on the local truncation error do not hold or become useless. Indeed, in certain instances a reduction in the order is observed (cf. [7] and the references therein).

2.4. The fully discrete approximations. Motivated by (2.8) and (2.9), we define the fully discrete approximations $\left\{u_{h}^{n}\right\}_{n=0}^{J}$ to the solution $u$ of (1.1) as follows: let $\pi_{h} u^{0}$ be any conveniently chosen element of $S_{h}^{r}$, e.g., $L^{2}$-projection, interpolant, etc. that satisfies

$$
\left\|u^{0}-\pi_{h} u^{0}\right\| \leq c h^{r} .
$$

Typically, the constant $c$ depends linearly on $\left\|u^{0}\right\|_{r}$. Let $u_{h}^{0}=\pi_{h} u^{0}$. For $n=0, \ldots, J-1$,

$$
\left(u_{h}^{n+1}, \chi\right)=\left(u_{h}^{n}, \chi\right)-k \sum_{i=1}^{q} b_{i}\left(u_{h}^{n, i} u_{h x}^{n, i}+u_{h x x x}^{n, i}, \chi\right) \quad \forall \chi \in S_{h}^{r},
$$

where

$$
\begin{array}{r}
\left(u_{h}^{n, i}, \chi\right)=\left(u_{h}^{n}, \chi\right)-k \sum_{j=1}^{q} a_{i j}\left(u_{h}^{n, j} u_{h x}^{n, j}+u_{h x x x}^{n, j}, \chi\right) \\
\forall \chi \in S_{h}^{r}, \quad i=1, \ldots, q .
\end{array}
$$

In [8], optimal, i.e., $O\left(k^{\nu}\right)$, rates of convergence were obtained by adding appropriate correction terms to the equations (2.14) in the case of two diagonally implicit methods with $\nu=3$ and $\nu=4$. In so doing, however, the schemes acquire a multistep nature, which places them outside the family of (single-step) RK methods. In a later paper [3], convergence rates of $p+2=q+2$ were obtained for the class of Gauss-Legendre methods [5]. The analysis, however, applies to other methods as well.

Using a different approach, we shall prove below that the full rate $\nu$ can indeed be obtained. In particular, for the Gauss-Legendre methods we have $\nu=$ $2 q$, the maximum for a given number $q$ of intermediate values. In addition, for these methods $m_{i j}=0$, which implies that $\left\|u_{h}^{n}\right\|=\left\|\pi_{h} u^{0}\right\|, n=0, \ldots, J$, a discrete analogue of the second conservation law of the $\mathrm{KdV}$ equation. 


\section{A SYSTEM OF NONLINEAR ORDINARY DIFFERENTIAL EQUATIONS}

In this section we show that the IRK methods applied to the solution of (1.1) at a given time $t^{n}$ yield a unique set of intermediate values $\left\{u^{n, i}\right\}_{i=1}^{q}$ given by

$$
u^{n, i}=u\left(t^{n}\right)-k \sum_{j=1}^{q} a_{i j}\left(u^{n, j} u_{x}^{n, j}+u_{x x x}^{n, j}\right), \quad i=1, \ldots, q,
$$

from which $u^{n+1}$ is given by

$$
u^{n+1}=u\left(t^{n}\right)-k \sum_{i=1}^{q} b_{i}\left(u^{n, i} u_{x}^{n, i}+u_{x x x}^{n, i}\right) .
$$

We also show that $\left\{u^{n, i}\right\}_{i=1}^{q}$ and $u^{n+1}$ possess the same degree of smoothness and periodicity as $u\left(t^{n}\right)$. Using this, in $\S 4$ we shall obtain the estimates $\left\|u^{n+1}-u\left(t^{n+1}\right)\right\|_{0, p} \leq c k^{\nu+1}, p=2, \infty$, where $t^{n+1}=t^{n}+k$.

Throughout this paper, we shall adopt the following notation: let $V=$ $\left(v_{1}, \ldots, v_{l}\right)^{T}$ and $W=\left(w_{1}, \ldots, w_{l}\right)^{T}$ be two $l$-tuples; by $V W$ we shall mean the $l$-tuple $\left(v_{1} w_{1}, \ldots, v_{l} w_{l}\right)^{T}$. For an $l \times l$ matrix $M, M V$ shall denote the $l$-tuple with $\sum_{j=1}^{l} M_{i j} v_{j}$ as $i$ th component. Also, $V_{x}$ shall denote $\left(v_{1 x}, \ldots, v_{l x}\right)^{T}$.

With this notation, we may express the equations (3.1) as

$$
U=e u\left(t^{n}\right)-k A U U_{x}-k A U_{x x x},
$$

where $U=\left(u^{n, 1}, \ldots, u^{n, q}\right)^{T}$ and $e u\left(t^{n}\right)=\left(u\left(t^{n}\right), \ldots, u\left(t^{n}\right)\right)^{T}$.

Some inequalities that will be used frequently are contained in the following

Lemma 3.1. (a) Let $v \in H^{1}$. Then

$$
\|v\|_{\infty}^{2} \leq\|v\|^{2}+2\|v\|\left\|v_{x}\right\| .
$$

(b) Let $v \in H^{2}$ be periodic. Then

$$
\left\|v_{x}\right\|_{\infty} \leq\left\|v_{x x}\right\| .
$$

(c) Let $v \in H^{3}$ be periodic. Then

$$
\begin{gathered}
\left\|v_{x}\right\| \leq\left\|v_{x x}\right\| \leq\left\|v_{x x x}\right\|, \\
\left\|v_{x}\right\|_{\infty}^{2} \leq\left\|v_{x}\right\|\left\|v_{x x}\right\| \leq\|v\|\left\|v_{x x x}\right\| .
\end{gathered}
$$

(d) Let $V, W \in \mathscr{H}^{3}$ be periodic. Then

$$
\left\|V W_{x}\right\|^{2} \leq\|V\|^{2}\|W\|\left\|W_{x x x}\right\| .
$$

Proof. (3.3) is standard; (3.4) and (3.5) are proved easily using integration by parts and periodicity. 
To prove (3.6), note that since $v$ is periodic, $v_{x}(a)=0$ for some $a \in[0,1]$. We may assume without loss of generality that $a=0$. Then, for $x \in[0,1]$,

$$
v_{x}^{2}(x)=2 \int_{0}^{x} v^{\prime}(t) v^{\prime \prime}(t) d t=-2 \int_{x}^{1} v^{\prime}(t) v^{\prime \prime}(t) d t .
$$

It follows that

$$
v_{x}^{2}(x) \leq \int_{0}^{1}\left|v^{\prime}(t)\right|\left|v^{\prime \prime}(t)\right| d t \leq\left\|v_{x}\right\|\left\|v_{x x}\right\| .
$$

To obtain the second inequality of (3.6), we integrate by parts to get

$$
\left\|v_{x}\right\|^{2}=-\int_{0}^{1} v(t) v^{\prime \prime}(t) d t \leq\|v\|\left\|v_{x x}\right\|
$$

Similarly,

$$
\left\|v_{x x}\right\|^{2}=-\int_{0}^{1} v^{\prime}(t) v^{\prime \prime \prime}(t) d t \leq\left\|v_{x}\right\|\left\|v_{x x x}\right\| .
$$

The desired inequality now follows by multiplying the last two inequalities, simplifying, and using the result in the first part of (3.6). To prove (3.7), we use (3.6) and the discrete Schwarz inequality:

$$
\begin{aligned}
\left\|V W_{x}\right\|^{2} & =\sum_{i=1}^{q}\left\|v_{i} w_{i x}\right\|^{2} \leq \sum_{i=1}^{q}\left\|v_{i}\right\|^{2}\left\|w_{i x}\right\|_{\infty}^{2} \leq\left(\sum_{i=1}^{q}\left\|v_{i}\right\|\left\|w_{i x}\right\|_{\infty}\right)^{2} \\
& \leq\|V\|^{2} \sum_{i=1}^{q}\left\|w_{i}\right\|\left\|w_{i x x x}\right\| \leq\|V\|^{2}\|W\|\left\|W_{x x x}\right\| .
\end{aligned}
$$

Lemma 3.2. Let $W \in \mathscr{C}^{m}, m \geq 0$, be periodic and assume $A$ satisfies (P). Then, for $k \neq 0$, the (linear) system

$$
V+k A V_{x x x}=W
$$

has a unique periodic solution in $\mathscr{C}^{m+3}$.

Proof. To show the existence of a unique solution to (3.8) in $\mathscr{C}^{m+3}$ with $V^{(j)}(0)=V^{(j)}(1), j=0, \ldots, m+2$, we need only show that the homogeneous system $V+k A V_{x x x}=0$ has no nontrivial $(\not \equiv 0)$ solution (cf. [9, Theorem 1.1, p. 408]). Indeed we get

$$
C(D V)+k D V_{x x x}=0,
$$

where $C=D A^{-1} D^{-1}$ is positive definite. Multiplying by $D V$ and integrating, we get $((C(D V), D V))=0$, which forces $D V \equiv 0$. Furthermore, writing $V_{x x x}=k^{-1} A^{-1}(W-V)$, we see that $V^{(m+3)}(0)=V^{(m+3)}(1)$.

Remark 3.1. In view of the continuous imbedding $\mathscr{H}^{m+1} \hookrightarrow \mathscr{C}^{m} \hookrightarrow \mathscr{H}^{m}$, $m \geq 0$, the solution $V$ of $(3.8)$ is periodic in $\mathscr{H}^{m+3}$ if $W$ is periodic in 
$\mathscr{H}^{m+1}$. Furthermore, from $V_{x x x}=k^{-1} A^{-1}(W-V)$, we see that $V$ is also periodic in $\mathscr{H}^{m+4}$.

Lemma 3.3. Let $f \in \mathscr{H}^{m+1}, U \in \mathscr{H}^{m}, m \geq 3$, be periodic and suppose that $A$ satisfies (P). Let the constants $M_{1}, M_{2}, M_{3}$, and $k_{0}$ be given by

$$
\begin{aligned}
& M_{1}=\frac{2 \alpha_{2}}{\alpha_{1}}\|D f\|, \\
& M_{2}=\frac{M_{1}^{3}}{\beta_{1}^{2} \gamma_{1}^{2}}\left(\beta_{2}+\frac{1}{\alpha_{1}}\right)^{2}+\frac{2}{\alpha_{1} \beta_{1}}\left\|D f_{x x x}\right\|, \\
& M_{3}=\frac{\left\|D f_{x x x x}\right\|}{\alpha_{1} \beta_{1}}+\frac{2}{\beta_{1} \gamma_{1}}\left(\beta_{2}+\frac{1}{\alpha_{1}}\right) M_{1} M_{2}, \\
& k_{0}=\frac{\alpha_{1} \gamma_{1}}{2 \sqrt{M_{1} M_{2}}} .
\end{aligned}
$$

Suppose that $0<k \leq k_{0},\|D U\| \leq M_{1}$, and $\left\|D U_{x x x}\right\| \leq M_{2}$. Then there exists a unique periodic solution $V$ in $\mathscr{H}^{m+1}$ to

$$
V+k A V_{x x x}=f-k A U U_{x}
$$

satisfying

$$
\left\{\begin{array}{l}
\|D V\| \leq M_{1}, \\
\left\|D V_{x x x}\right\| \leq M_{2}, \\
\left\|D V_{x x x x}\right\| \leq M_{3} .
\end{array}\right.
$$

Proof. Since $U \in \mathscr{H}^{m}$, it follows that $U \in \mathscr{C}^{m-1}$; hence $U U_{x} \in \mathscr{C}^{m-2} \subseteq$ $\mathscr{H}^{m-2}$. Thus, by Lemma 3.2 and Remark 3.1, there exists a unique periodic $V \in \mathscr{H}^{m+1}$ satisfying (3.10). To prove the first estimate in (3.11), we rewrite (3.10) as

$$
C(D V)+k D V_{x x x}=C D f-k D U U_{x},
$$

from which it follows that

$$
((C(D V), D V))+k\left(\left(D V_{x x x}, D V\right)\right)=((C D f, D V))-k\left(\left(D U U_{x}, D V\right)\right)
$$

and thus, by periodicity and $(\mathrm{P})$,

$$
\alpha_{1}\|D V\|^{2} \leq \alpha_{2}\|D f\|\|D V\|+k\left\|D U U_{x}\right\|\|D V\|,
$$

and hence

Now using (3.7),

$$
\|D V\| \leq \frac{\alpha_{2}}{\alpha_{1}}\|D f\|+\frac{k}{\alpha_{1}}\left\|D U U_{x}\right\|
$$

$$
\begin{aligned}
\|D V\| & \leq \frac{M_{1}}{2}+\frac{k}{\alpha_{1} \gamma_{1}}\|D U\|^{3 / 2}\left\|D U_{x x x}\right\|^{1 / 2} \\
& \leq \frac{M_{1}}{2}+\frac{k M_{1}^{3 / 2} M_{2}^{1 / 2}}{\alpha_{1} \gamma_{1}} \leq M_{1} .
\end{aligned}
$$


Also $V-f=-k A V_{x x x}-k A U U_{x}$. Hence

$$
\begin{aligned}
((C D(V-f), D(V-f)))= & k\left(\left(D V_{x x x}, D(f-V)\right)\right) \\
& +k\left(\left(D U U_{x}, D(f-V)\right)\right) .
\end{aligned}
$$

So

$$
\alpha_{1}\|D(V-f)\|^{2} \leq k\left\|D U U_{x}\right\|\|D(f-V)\|+k\left(\left(D V_{x x x}, D f\right)\right) .
$$

Moreover,

$$
\left(\left(D V_{x x x}, D f\right)\right)=-\left(\left(D V, D f_{x x x}\right)\right)=\left(\left(D(f-V), D f_{x x x}\right)\right) .
$$

Hence,

$$
\alpha_{1}\|D(V-f)\| \leq k\left(\left\|D U U_{x}\right\|+\left\|D f_{x x x}\right\|\right) .
$$

From (3.10),

$$
k C^{-1} D V_{x x x}=D(f-V)-k C^{-1} D U U_{x} .
$$

Hence, from (3.12) and (3.7),

$$
\begin{aligned}
\beta_{1}\left\|D V_{x x x}\right\| & \leq \frac{1}{k}\|D(f-V)\|+\beta_{2}\left\|D U U_{x}\right\| \\
& \leq \frac{1}{\alpha_{1}}\left\|D f_{x x x}\right\|+\left(\beta_{2}+\frac{1}{\alpha_{1}}\right)\left\|D U U_{x}\right\| \\
& \leq \frac{1}{\alpha_{1}}\left\|D f_{x x x}\right\|+\left(\beta_{2}+\frac{1}{\alpha_{1}}\right) \frac{M_{1}^{3 / 2} M_{2}^{1 / 2}}{\gamma_{1}} .
\end{aligned}
$$

Hence,

$$
\left\|D V_{x x x}\right\| \leq \frac{1}{\alpha_{1} \beta_{1}}\left\|D f_{x x x}\right\|+\frac{1}{2}\left(\beta_{2}+\frac{1}{\alpha_{1}}\right)^{2} \frac{M_{1}^{3}}{\gamma_{1}^{2} \beta_{1}^{2}}+\frac{M_{2}}{2}=M_{2} .
$$

To estimate $\left\|D V_{x x x x}\right\|$, differentiating (3.10) and proceeding as above, we obtain

$$
\left\|D V_{x x x x}\right\| \leq \frac{1}{\alpha_{1} \beta_{1}}\left\|D f_{x x x x}\right\|+\frac{1}{\beta_{1}}\left(\beta_{2}+\frac{1}{\alpha_{1}}\right)\left\|\left(D U U_{x}\right)_{x}\right\| .
$$

Now

$$
\begin{aligned}
\left\|\left(D U U_{x}\right)_{x}\right\|^{2} & =\sum_{i=1}^{q}\left\|d_{i}\left(u_{i} u_{i x}\right)_{x}\right\|^{2} \leq \sum_{i=1}^{q}\left(\left\|d_{i} u_{i} u_{i x x}\right\|+\left\|d_{i} u_{i x} u_{i x}\right\|\right)^{2} \\
& \leq \sum_{i=1}^{q}\left(\left\|d_{i} u_{i}\right\|\left\|u_{i x x}\right\|_{\infty}+\left\|d_{i} u_{i x}\right\|_{\infty}\left\|u_{i x}\right\|_{\infty}\right)^{2} .
\end{aligned}
$$

Then, using (3.4), (3.5), and (3.6), we get

$$
\left\|\left(D U U_{x}\right)_{x}\right\| \leq 2\|D U\|\left\|U_{x x x}\right\| \leq \frac{2}{\gamma_{1}}\|D U\|\left\|D U_{x x x}\right\| \leq \frac{2}{\gamma_{1}} M_{1} M_{2} .
$$


Hence,

$$
\left\|D V_{x x x x}\right\| \leq \frac{1}{\alpha_{1} \beta_{1}}\left\|D f_{x x x x}\right\|+\frac{2}{\beta_{1} \gamma_{1}}\left(\beta_{2}+\frac{1}{\alpha_{1}}\right) M_{1} M_{2}=M_{3} .
$$

This concludes the proof.

We are now in a position to prove the following

Theorem 3.1. Let $f \in \mathscr{H}^{m+1}, m \geq 3$, be periodic. Suppose $A$ satisfies (P), and let the constants $M_{1}, M_{2}, M_{3}, k_{0}$ be as in Lemma 3.3. There exists a constant $0<k_{1} \leq k_{0}$ such that if $k \leq k_{1}$, then there exists a periodic solution $\dot{U}$ in $\mathscr{H}^{m+1}$ to

$$
U+k A U U_{x}+k A U_{x x x}=f
$$

satisfying

$$
\|U\|_{m+1} \leq c=c\left(\|f\|_{m+1}\right), \quad m \geq 3 .
$$

Furthermore, there exists a constant $0<k_{2} \leq k_{0}$ such that if $k \leq k_{2}$, then the solution $U$ of (3.15) is unique.

Proof. Consider the sequence $\left\{U_{j}\right\}_{j \geq 0}$ generated by

$$
\left\{\begin{array}{l}
U_{j+1}+k A U_{j+1, x x x}=f-k A U_{j} U_{j x}, \quad j=0,1, \ldots, \\
U_{0} \equiv 0 .
\end{array}\right.
$$

Note that trivially $U_{0}$ is periodic in $\mathscr{H}^{m+1},\left\|D U_{0}\right\| \leq M_{1}$, and $\left\|D U_{0 x x x}\right\| \leq$ $M_{2}$; by Lemma 3.3 and Remark 3.1, $U_{1}$ exists uniquely and is periodic in $\mathscr{H}^{m+1}$. Furthermore, $\left\|D U_{1}\right\| \leq M_{1}$ and $\left\|D U_{1 x x x}\right\| \leq M_{2}$. This argument may be used repeatedly to yield a well-defined sequence $\left\{U_{j}\right\}_{j \geq 0}$. For $j \geq 0, U_{j}$ is periodic in $\mathscr{H}^{m+1}$ and satisfies $\left\|D U_{j x x x x}\right\| \leq M_{3}$.

Now if $U_{j^{*}}=U_{j^{*}+1}$ for some $j^{*} \geq 0$, then $U_{j^{*}}$ is a solution of (3.15), since $U_{j^{*}} \in \mathscr{H}^{4} \subseteq \mathscr{C}^{3}$. Otherwise, the sequence $\left\{U_{j}\right\}_{j \geq 0}$ is infinite and is bounded in $\mathscr{H}^{4}$. Since $\mathscr{H}^{4}$ is compactly imbedded in $\mathscr{C}^{3}$, we can extract a subsequence $\left\{U_{j^{\prime}}\right\}$ which converges in $\mathscr{C}^{3}$ to some $U$. The latter must indeed satisfy (3.15). To see this, it is sufficient to show that $\left\{U_{j}\right\}_{j=0}^{\infty}$ is Cauchy in $\mathscr{H}^{1}$. Indeed, from previously used techniques, it follows that

$$
\left\|U_{j+1}-U_{j}\right\|_{1} \leq c k^{1 / 2}\left\|U_{j}-U_{j-1}\right\|_{1}, \quad j=1,2, \ldots,
$$

for some $c=c\left(\|f\|_{3}\right)$. Thus, the map $U_{j} \rightarrow U_{j+1}$ is a contraction in $\mathscr{H}^{1}$ for $k$ sufficiently small.

To show uniqueness, assume the existence of two solutions $U_{1}$ and $U_{2}$ of (3.15) and let $E=U_{1}-U_{2}$. Then

$$
E+k A E_{x x x}=-k A E U_{2 x}-k A E_{x} U_{1},
$$

from which we obtain

$$
((C D E, D E))=-k\left\{\left(\left(D E U_{2 x}, D E\right)\right)-\frac{1}{2}\left(\left(D E U_{1 x}, D E\right)\right)\right\} .
$$


Hence

$$
\alpha_{1}\|D E\|^{2} \leq k\left(\left\|U_{2 x}\right\|_{\infty}+\left\|U_{1 x}\right\|_{\infty}\right)\|D E\|^{2} .
$$

Uniqueness will follow, for $k$ sufficiently small, from the estimate

$$
\left\|U_{x}\right\|_{\infty} \leq c=c\left(\|f\|_{3}\right)
$$

for periodic solutions $U$ of (3.15). Indeed, rewriting (3.15) as

$$
C D U+k D U U_{x}+k D U_{x x x}=C D f,
$$

multiplying by $D U$ and integrating, we first obtain the estimate

$$
\|U\| \leq c\|f\| \text {. }
$$

Now letting $V=U$ in (3.13) and using (3.7), we obtain

$$
\left\|U_{x x x}\right\| \leq c\left\|f_{x x x}\right\|+c\left\|U U_{x}\right\| \leq c\left\|f_{x x x}\right\|+c\|U\|^{3 / 2}\left\|U_{x x x}\right\|^{1 / 2} ;
$$

from this, and using (3.18), it follows that $\left\|U_{x x x}\right\| \leq c\left(\|f\|_{3}\right) ;(3.17)$ now follows from (3.18) and (3.6).

We now establish (3.16). From (3.11) it follows that $\|U\|_{l} \leq c\left(\|f\|_{l}\right), l=$ $0,3,4$. So, as an induction hypothesis assume that

$$
\|U\|_{l} \leq c\left(\|f\|_{l}\right), \quad l=3, \ldots, s \leq m .
$$

We next prove that as a consequence of (3.19),

$$
\|f-U\|_{j} \leq k c\left(\|f\|_{j+3}\right), \quad j=0, \ldots, s-2 .
$$

Differentiating (3.15) $j$ times, we get

$$
C D(U-f)^{(j)}=-k D\left(U U_{x}\right)^{(j)}-k D U^{(j+3)} .
$$

Multiplying by $D(U-f)^{(j)}$ and integrating, we get

$$
\begin{aligned}
\left(\left(C D(U-f)^{(j)}, D(U-f)^{(j)}\right)\right)= & -k\left(\left(D\left(U U_{x}\right)^{(j)}, D(U-f)^{(j)}\right)\right) \\
& -k\left(\left(D(U-f)^{(j)}, D f^{(j+3)}\right)\right) .
\end{aligned}
$$

Hence,

$$
\left\|(U-f)^{(j)}\right\| \leq c k\left\|D\left(U U_{x}\right)^{(j)}\right\|+c k\left\|D f^{(j+3)}\right\| .
$$

Using Leibniz' formula, from (3.3) and (3.19) it follows that

$$
\begin{aligned}
\left\|D\left(U U_{x}\right)^{(j)}\right\| & \leq c \sum_{k=0}^{j}\left\|U^{(k)}\right\|_{\infty}\left\|U^{(j-k+1)}\right\| \\
& \leq c\left(\|f\|_{j+1}\right), \quad j=0, \ldots, s-2 ;
\end{aligned}
$$

(3.20) now follows from (3.22) and (3.23).

Again differentiating (3.15) $s-2$ times, we get

$$
C^{-1} D U^{(s+1)}=\frac{1}{k} D(f-U)^{(s-2)}-C^{-1} D\left(U U_{x}\right)^{(s-2)} \text {. }
$$


Multiplying by $D U^{(s+1)}$ and integrating, we get

$$
\left\|U^{(s+1)}\right\| \leq \frac{c}{k}\left\|(f-U)^{(s-2)}\right\|+c\left\|\left(U U_{x}\right)^{(s-2)}\right\| .
$$

Using (3.20), (3.23) in (3.24), we get

$$
\left\|U^{(s+1)}\right\| \leq c\left(\|f\|_{s+1}\right) .
$$

This concludes the induction argument and the proof of the theorem.

In the sequel, we shall take $f=e u\left(t^{n}\right)$. It follows from (3.2) that $u^{n+1}=$ $\left(1-b^{T} A^{-1} e\right) u\left(t^{n}\right)+b^{T} A^{-1} U$. In view of the estimates (3.16) and the a priori estimates on the solution $u$ of (1.1), we have

$$
\left\|u^{n+1}\right\|_{m},\|U\|_{m} \leq c\left(\left\|u^{0}\right\|_{m}\right), \quad m \geq 4 .
$$

\section{CONSISTENCY, STABILITY, AND CONVERGENCE}

In estimating the local truncation error $\left\|u\left(t^{n+1}\right)-u^{n+1}\right\|$, we introduce the set of functions $\left\{\alpha_{i j}(x)\right\}, i=1, \ldots, q, j=0, \ldots, \nu$, defined recursively by

$$
\left\{\begin{aligned}
& \alpha_{i 0}=u\left(t^{n}\right), \quad i=1, \ldots, q, \\
& \alpha_{i, l+1}=-\sum_{j=1}^{q} a_{i j}\left\{\sum_{m=0}^{l} \alpha_{j m} \alpha_{j, l-m}^{\prime}+\alpha_{j l}^{\prime \prime \prime}\right\}, \\
& i=1, \ldots, q, l=0, \ldots, \nu-1 .
\end{aligned}\right.
$$

Here $\left({ }^{\prime}\right)$ denotes differentiation with respect to $x$.

Note that if $u\left(t^{n}\right) \in C^{m}$ is periodic with $m \geq 3 \nu$, then $\alpha_{i j}$ is periodic in $C^{m-3 j}$ for $i=1, \ldots, q, j=0, \ldots, \nu$.

Lemma 4.1. Assume that $(\mathrm{C})$ holds together with (2.10b). Denoting the vector $\left(\alpha_{1 j}, \ldots, \alpha_{q j}\right)^{T}$ by $\alpha_{j}$, with $D_{t}^{j} u=\left.\left(\partial^{j} / \partial t^{j}\right) u(x, t)\right|_{t=t^{n}}$ we have

$$
\begin{gathered}
\alpha_{j}=\frac{T^{j} e}{j !} D_{t}^{j} u, \quad j=0, \ldots, p, \quad \text { if } p \leq \nu, \\
\alpha_{p+1}=\frac{A T^{p} e}{p !} D_{t}^{p+1} u, \quad \text { if } p \leq \nu-1, \\
\alpha_{l+1}=\frac{A T^{l} e}{l !} D_{t}^{l+1} u \\
-\sum_{m=p+1}^{l} A\left[\frac{T^{l-m}}{(l-m) !} \alpha_{m}-\frac{T^{l} e}{m !(l-m) !} D_{t}^{m} u\right] D_{t}^{l-m} u^{\prime} \\
-\sum_{m=0}^{p} A\left[\frac{T^{m}}{m !} \alpha_{l-m}^{\prime}-\frac{T^{l} e}{m !(l-m) !} D_{t}^{l-m} u^{\prime}\right] D_{t}^{m} u \\
-A\left[\alpha_{l}^{\prime \prime \prime}-\frac{T^{l} e}{l !} D_{t}^{l} u^{\prime \prime \prime}\right], \quad l=p+1, \ldots, \nu-1, \quad \text { if } p \leq \nu-2 .
\end{gathered}
$$


Proof. Obviously, (4.2) holds with $j=0$. Suppose it also holds for $j=$ $0, \ldots, l \leq p-1$. Then, using Leibniz' formula,

$$
\begin{aligned}
\alpha_{i, l+1} & =-\sum_{j=1}^{q} a_{i j}\left\{\sum_{m=0}^{l} \frac{\tau_{j}^{m}}{m !} D_{t}^{m} u \frac{\tau_{j}^{l-m}}{(l-m) !} D_{t}^{l-m} u^{\prime}+\frac{\tau_{j}^{l}}{l !} D_{t}^{l} u^{\prime \prime \prime}\right\} \\
& =-\frac{1}{l !} \sum_{j=1}^{q} a_{i j} \tau_{j}^{l}\left\{D_{t}^{l}\left(u u^{\prime}\right)+D_{t}^{l} u^{\prime \prime \prime}\right\}=\frac{D_{t}^{l+1} u}{l !} \sum_{j=1}^{q} a_{i j} \tau_{j}^{l} \\
& =\frac{\tau_{i}^{l+1}}{(l+1) !} D_{t}^{l+1} u, \quad i=1, \ldots, q,
\end{aligned}
$$

using (1.1) and (C). We also obtain (4.3) in a similar manner.

Now using (4.2) and Leibniz' formula, in view of $\nu \leq 2 p+2$,

$$
\begin{aligned}
& \alpha_{i, l+1}=-\sum_{j=1}^{q} a_{i j}\left\{\sum_{m=p+1}^{l} \alpha_{j m} \frac{\tau_{j}^{l-m}}{(l-m) !} D_{t}^{l-m} u^{\prime}\right. \\
&\left.+\sum_{m=0}^{p} \frac{\tau_{j}^{m}}{m !} D_{t}^{m} u \alpha_{j, l-m}^{\prime}+\alpha_{j l}^{\prime \prime \prime}\right\} \\
&=-\sum_{j=1}^{q} a_{i j}\left\{\sum_{m=0}^{l} \frac{\tau_{j}^{m}}{m !} D_{t}^{m} u \frac{\tau_{j}^{l-m}}{(l-m) !} D_{t}^{l-m} u^{\prime}+\frac{\tau_{j}^{l}}{l !} D_{t}^{l} u^{\prime \prime \prime}\right. \\
&+\sum_{m=p+1}^{l}\left[\alpha_{j m}-\frac{\tau_{j}^{m}}{m !} D_{t}^{m} u\right] \frac{\tau_{j}^{l-m}}{(l-m) !} D_{t}^{l-m} u^{\prime} \\
&+\sum_{m=0}^{p} \frac{\tau_{j}^{m}}{m !} D_{t}^{m} u\left[\alpha_{j, l-m}^{\prime}-\frac{\tau_{j}^{l-m}}{(l-m) !} D_{t}^{l-m} u^{\prime}\right] \\
&\left.+\left[\alpha_{j l}^{\prime \prime \prime}-\frac{\tau_{j}^{l}}{l !} D_{t}^{l} u^{\prime \prime \prime}\right]\right\} .
\end{aligned}
$$

Now (4.4) follows from (1.1).

As we shall see below, the $\alpha$ 's are the coefficients of the expansions of the intermediate stages $u^{n, i}$ in powers of $k$, up to order $\nu$. In view of (C), the $\alpha$ 's are, up to order $p+1$, time-derivatives of $u$ at $t=t^{n}$. This ceases to be the case from $p+2$ to $\nu$. However, using (B) and (D), it will be shown below that appropriate linear combinations of the $\alpha$ 's are again time-derivatives of $u$. We make this precise in the following

Lemma 4.2. Assume that (B), (C), (D), (2.10a), and (2.10b) hold. Then for each $l=0, \ldots, \nu-1$,

$$
b^{T} T^{s} \alpha_{l}=\frac{D_{t}^{l} u}{l !(s+l+1)} \quad \forall s=0, \ldots, \nu-1 \text { with } s+l \leq \nu-1 .
$$


Proof. Assume $0 \leq l \leq p, 0 \leq s \leq \nu-1$ with $s+l \leq \nu-1$. From (4.2) and (B),

$$
b^{T} T^{s} \alpha_{l}=b^{T} T^{s} \frac{T^{l} e}{l !} D_{t}^{l} u=b^{T} \frac{T^{s+l} e}{l !} D_{t}^{l} u=\frac{D_{t}^{l} u}{l !(s+l+1)} .
$$

Now let $l=p+1$ and $0 \leq s \leq \nu-1$ with $s+p+1 \leq \nu-1$. It follows from the inequalities $\nu \leq r+p+1$ and $s+p+1 \leq \nu-1$ that $s \leq r-1$; hence, using (B) and (D), we obtain from (4.3)

$$
\begin{aligned}
b^{T} T^{s} \alpha_{p+1} & =\frac{b^{T} T^{s} A T^{p} e}{p !} D_{t}^{p+1} u=\frac{1}{p !} \frac{1}{s+1} b^{T}\left(I-T^{s+1}\right) T^{p} e D_{t}^{p+1} u \\
& =\frac{1}{(s+1) p !}\left\{b^{T} T^{p} e-b^{T} T^{s+1+p} e\right\} D_{t}^{p+1} u \\
& =\frac{1}{(s+1) p !}\left\{\frac{1}{p+1}-\frac{1}{s+p+2}\right\} D_{t}^{p+1} u \\
& =\frac{D_{t}^{p+1} u}{(p+1) !(s+p+2)}
\end{aligned}
$$

We now complete the proof using an induction argument: assume (4.5) holds up to some $l$, with $p+1 \leq l \leq \nu-2$. From (4.4) we have for $s=0, \ldots, \nu-1$ with $s+l+1 \leq \nu-1$,

$$
\begin{aligned}
b^{T} T^{s} \alpha_{l+1}= & \frac{b^{T} T^{s} A T^{l} e}{l !} D_{t}^{l+1} u \\
& -\sum_{m=p+1}^{l} b^{T} T^{s} A\left[\frac{T^{l-m}}{(l-m) !} \alpha_{m}-\frac{T^{l} e}{m !(l-m) !} D_{t}^{m} u\right] D_{t}^{l-m} u^{\prime} \\
& -\sum_{m=0}^{p} b^{T} T^{s} A\left[\frac{T^{m}}{m !} \alpha_{l-m}^{\prime}-\frac{T^{l} e}{m !(l-m) !} D_{t}^{l-m} u^{\prime}\right] D_{t}^{m} u \\
& -b^{T} T^{s} A\left[\alpha_{l}^{\prime \prime \prime}-\frac{T^{l} e}{l !} D_{t}^{l} u^{\prime \prime \prime}\right] .
\end{aligned}
$$

As before, $s \leq r-1$. Hence, using (D) and (B),

$$
\begin{aligned}
b^{T} T^{s} A T^{l} e & =\frac{b^{T}}{s+1}\left(I-T^{s+1}\right) T^{l} e \\
& =\frac{1}{s+1}\left\{b^{T} T^{l} e-b^{T} T^{s+1+l} e\right\}=\frac{1}{(l+1)(s+l+2)}
\end{aligned}
$$

thus

$$
\frac{b^{T} T^{s} A T^{l} e}{l !} D_{t}^{l+1} u=\frac{D_{t}^{l+1} u}{(l+1) !(s+l+2)} .
$$

The result will now follow from the fact that the second, third, and fourth terms on the right side of (4.6) vanish. We consider only the second term. From 
(D) and (B), for $p+1 \leq m \leq l$,

$$
\begin{aligned}
b^{T} T^{s} A T^{l-m} \alpha_{m} & =\frac{b^{T}}{s+1}\left(I-T^{s+1}\right) T^{l-m} \alpha_{m} \\
& =\frac{1}{s+1}\left\{b^{T} T^{l-m} \alpha_{m}-b^{T} T^{s+l-m+1} \alpha_{m}\right\} \\
& =\frac{1}{s+1}\left\{\frac{D_{t}^{m} u}{m !(l+1)}-\frac{D_{t}^{m} u}{m !(s+l+2)}\right\} \\
& =\frac{D_{t}^{m} u}{m !(l+1)(s+l+2)} .
\end{aligned}
$$

As before,

$$
b^{T} T^{s} A T^{l} e=\frac{1}{(l+1)(s+l+2)} .
$$

So indeed the second term is zero.

We have

Corollary 4.1. Under the conditions of Lemmas 4.1 and 4.2,

$$
b^{T} A^{-1} \alpha_{l}=\frac{D_{t}^{l} u}{l !}, \quad l=1, \ldots, \nu .
$$

Proof. For $l=1, \ldots, p$, using (C) and (B) and (4.2), we get

$$
b^{T} A^{-1} \alpha_{l}=b^{T} A^{-1} \frac{T^{l} e}{l !} D_{t}^{l} u=b^{T} A^{-1} \frac{A T^{l-1} e}{(l-1) !} D_{t}^{l} u=\frac{D_{t}^{l} u}{l !}
$$

For $l=p+1$, from (4.3) and (B),

$$
b^{T} A^{-1} \alpha_{p+1}=\frac{b^{T} T^{p} e}{p !} D_{t}^{p+1} u=\frac{D_{t}^{p+1} u}{(p+1) !} .
$$

For $l=p+1, \ldots, \nu-1$, we get from (4.4)

$$
\begin{aligned}
b^{T} A^{-1} \alpha_{l+1}= & \frac{b^{T} T^{l} e}{l !} D_{t}^{l+1} u \\
& -\sum_{m=p+1}^{l} b^{T}\left[\frac{T^{l-m}}{(l-m) !} \alpha_{m}-\frac{T^{l} e}{m !(l-m) !} D_{t}^{m} u\right] D_{t}^{l-m} u^{\prime} \\
& -\sum_{m=0}^{p} b^{T}\left[\frac{T^{m}}{m !} \alpha_{l-m}^{\prime}-\frac{T^{l} e}{m !(l-m) !} D_{t}^{l-m} u^{\prime}\right] D_{t}^{m} u \\
& -b^{T}\left[\alpha_{l}^{\prime \prime \prime}-\frac{T^{l} e}{l !} D_{t}^{l} u^{\prime \prime \prime}\right] .
\end{aligned}
$$

Using (B), the first term on the right side gives $D_{t}^{l+1} u /(l+1) !$. On the other hand, the second, third, and fourth terms vanish in view of (4.5) and (B).

We now consider briefly the special case of the 3-stage diagonally implicit method, for which, as we recall, $2 p+2=4$ but $r+p+1=3$. We need only 
verify that $b^{T} A^{-1} \alpha_{4}=D_{t}^{4} u / 24$. That this identity indeed holds can be seen by using Lemma 4.1 in conjunction with the following three identities:

$$
b^{T} T A T e=1 / 8, \quad b^{T} A T^{2} e=1 / 12, \quad b^{T} A^{2} T e=1 / 24 .
$$

In the next result we estimate the local truncation error.

Theorem 4.1. Suppose that (3.25) holds with $m \geq 3 \nu+4$. Suppose also that (B), (C), (D), (P), and (4.8) hold. Then there exists $k^{*}>0$ such that if $k<k^{*}$,

$$
\left\|u\left(t^{n+1}\right)-u^{n+1}\right\|_{0, p} \leq c k^{\nu+1}, \quad p=2 \text { and } p=\infty,
$$

where $u^{n+1}$ is given by (3.2) and $c$ is a constant independent of $k$.

Proof. Let $U=\left(u^{n, 1}, \ldots, u^{n, q}\right)^{T}$ and $\left\{\alpha_{i j}\right\}, i=1, \ldots, q, j=0, \ldots, \nu$, be given by (3.1) and (4.1), respectively. Let $e^{n, i}$ be given by

$$
u^{n, i}=\sum_{l=0}^{\nu} k^{l} \alpha_{i l}+e^{n, i}, \quad i=1, \ldots, q .
$$

Using (4.10) in (3.1), we get for $i=1, \ldots, q$

$$
\begin{aligned}
& \sum_{l=0}^{\nu} k^{l} \alpha_{i l}+e^{n, i} \\
& =u\left(t^{n}\right)-k \sum_{j=1}^{q} a_{i j}\left\{\left(\sum_{l=0}^{\nu} k^{l} \alpha_{j l}+e^{n, j}\right)\left(\sum_{l=0}^{\nu} k^{l} \alpha_{j l}+e^{n, j}\right)^{\prime}\right. \\
& \left.+\left(\sum_{l=0}^{\nu} k^{l} \alpha_{j l}+e^{n, j}\right)^{\prime \prime \prime}\right\} \\
& =u\left(t^{n}\right)-k \sum_{j=1}^{q} a_{i j}\left\{\sum_{l=0}^{\nu-1} k^{l} \sum_{m=0}^{l} \alpha_{j_{m}} \alpha_{j, l-m}^{\prime}\right. \\
& +k^{\nu} \sum_{0 \leq m_{1}, m_{2} \leq \nu} c_{j m_{1} m_{2}} \alpha_{j m_{1}} \alpha_{j m_{2}}^{\prime}+\sum_{l=0}^{\nu} k^{l} \alpha_{j l}^{\prime \prime \prime} \\
& \left.+\left[\left(\sum_{l=0}^{\nu} k^{l} \alpha_{j l}\right) e^{n, j}+\frac{1}{2}\left(e^{n, j}\right)^{2}\right]^{\prime}+\left(e^{n, j}\right)^{\prime \prime \prime}\right\}
\end{aligned}
$$

for some constants $c_{j m_{1} m_{2}}$ that are bounded from above independently of $k$. Now using (4.1), for $i=1, \ldots, q$,

$$
\begin{aligned}
e^{n, i}=-k \sum_{j=1}^{q} a_{i j}\left\{k^{\nu}\left(\sum_{0 \leq m_{1}, m_{2} \leq \nu} c_{j m_{1} m_{2}} \alpha_{j m_{1}} \alpha_{j m_{2}}^{\prime}+\alpha_{j \nu}^{\prime \prime \prime}\right)\right. \\
\left.+\left[\left(\sum_{l=0}^{\nu} k^{l} \alpha_{j l}\right) e^{n, j}+\frac{1}{2}\left(e^{n, j}\right)^{2}\right]^{\prime}+\left(e^{n, j}\right)^{\prime \prime \prime}\right\} .
\end{aligned}
$$


We rewrite $(4.11)$ as

$$
C D E=-k^{\nu+1} D \Psi-k D(\Phi E)_{x}-\frac{k}{2} D\left(E^{2}\right)_{x}-k D E_{x x x}
$$

with

$$
\begin{gathered}
E=\left(e^{n, 1}, \ldots, e^{n, q}\right)^{T}, \quad \Phi=\sum_{l=0}^{\nu} k^{l} \alpha_{l}=U-E, \\
\Psi_{i}=\sum_{0 \leq m_{1}, m_{2} \leq \nu} c_{i m_{1} m_{2}} \alpha_{i m_{1}} \alpha_{i m_{2}}^{\prime}+\alpha_{i \nu}^{\prime \prime \prime} .
\end{gathered}
$$

Multiplying by $D E$, integrating, and using periodicity, we get

$$
((C D E, D E))=-k^{\nu+1}((D \Psi, D E))-\frac{k}{2}\left(\left(D U_{x}, D E^{2}\right)\right),
$$

from which we obtain the estimate

$$
\|E\|^{2} \leq c k^{\nu+1}\|\Psi\|\|E\|+c k\left\|U_{x}\right\|_{\infty}\|E\|^{2} .
$$

Using (4.1) and an induction argument, it is easily proved that

$$
\alpha_{i l}=c_{i l} u^{(3 l)}+P_{i l}\left(u^{(0)}, \ldots, u^{(3 l-2)}\right), \quad i=1, \ldots, q, l=0, \ldots, \nu
$$

where $c_{i l}$ is a constant, $P_{i l}$ is a polynomial in $3 l-1$ variables, and $u^{(j)}=$ $\left.D_{x}^{j} u\right|_{t=t^{n}}$. Using (4.14), the definition of $\Psi,(3.3)$, and (3.4), we get $\|\Psi\| \leq$ $c\left(\left\|u^{0}\right\|_{3 \nu+3}\right)$. Using this and (3.17) in (4.13), for $k$ sufficiently small we get

$$
\|E\| \leq c k^{\nu+1} \text {. }
$$

Now from (3.1), (3.2), (4.8), and (4.10),

$$
\begin{aligned}
u^{n+1} & =u\left(t^{n}\right)-k b^{T}\left(U U_{x}+U_{x x x}\right)=u\left(t^{n}\right)+b^{T} A^{-1}\left(U-e u\left(t^{n}\right)\right) \\
& =u\left(t^{n}\right)+\sum_{l=1}^{\nu} k^{l} b^{T} A^{-1} \alpha_{l}+b^{T} A^{-1} E=\sum_{l=0}^{\nu} k^{l} \frac{D_{t}^{l} u}{l !}+b^{T} A^{-1} E .
\end{aligned}
$$

Expanding $u\left(t^{n+1}\right)$ in a Taylor series at $t^{n}$, we see that

$$
u^{n+1}-u\left(t^{n+1}\right)=-\frac{1}{\nu !} \int_{t^{n}}^{t^{n+1}}\left(t^{n+1}-s\right)^{\nu} D_{t}^{\nu+1} u(s) d s+b^{T} A^{-1} E
$$

it follows from (4.15) and (4.16) that

$$
\left\|u^{n+1}-u\left(t^{n+1}\right)\right\| \leq c k^{\nu+1} .
$$

Now multiplying (4.12) by $-D E_{x x}-\frac{1}{2} D E^{2}$ and integrating, we get

$$
\begin{aligned}
\left(\left(C D E_{x}, D E_{x}\right)\right)= & \frac{1}{2}\left(\left(C D E, D E^{2}\right)\right)-k^{\nu+1}\left(\left(D \Psi_{x}, D E_{x}\right)\right) \\
& +\frac{1}{2} k^{\nu+1}\left(\left(D \Psi, D E^{2}\right)\right)+\frac{k}{2}\left(\left(D \Phi_{x x x}, D E^{2}\right)\right) \\
& -\frac{3 k}{2}\left(\left(D \Phi_{x}, D\left(E_{x}\right)^{2}\right)\right)+\frac{k}{3}\left(\left(D \Phi_{x}, D E^{3}\right)\right) .
\end{aligned}
$$


From this it follows that

$$
\begin{aligned}
\left\|E_{x}\right\|^{2} \leq & c\|E\|_{\infty}\|E\|^{2}+c k^{\nu+1}\left\|\Psi_{x}\right\|\left\|E_{x}\right\|+c k^{\nu+1}\|\Psi\|_{\infty}\|E\|^{2} \\
& +c k\left\|\Phi_{x x x}\right\|_{\infty}\|E\|^{2}+c k\left\|\Phi_{x}\right\|_{\infty}\left\|E_{x}\right\|^{2} \\
& +c k\left\|\Phi_{x}\right\|_{\infty}\|E\|_{\infty}\|E\|^{2} .
\end{aligned}
$$

Now using (4.14), (3.3), (3.4), and the definition of $\Phi, \Psi$, it follows that $\left\|\Psi_{x}\right\|,\|\Psi\|_{\infty},\left\|\Phi_{x x x}\right\|_{\infty},\left\|\Phi_{x}\right\|_{\infty} \leq c\left(\left\|u^{0}\right\|_{3 \nu+4}\right)$; hence, using (4.15), it follows from (4.18) that

$$
\left\|E_{x}\right\|^{2} \leq c k^{2 \nu+2}+c k^{2 \nu+2}\|E\|_{\infty} .
$$

Using (3.3) and (4.15), it follows that

$$
\|E\|_{\infty} \leq\|E\|+\left\|E_{x}\right\| \leq c k^{\nu+1} .
$$

(4.9) now follows from (4.15), (4.16), and (4.19).

In the sequel, we shall let $u^{n}=u\left(t^{n}\right)$, and $u^{n+1}, u^{n, i}, i=1, \ldots, q$, be as in (3.1), (3.2). Let $U^{n+1}, U^{n}, U^{n, i}, U^{n, i} U_{x}^{n, i}, U_{x x x}^{n, i}$ denote the $N$-vectors whose $j$ th components are $u^{n+1}(j h), u^{n}(j h), u^{n, i}(j h), u^{n, i}(j h) u_{x}^{n, i}(j h)$, and $u_{x x x}^{n, i}(j h)$, respectively.

Also, let $u_{h}^{n}, u_{h}^{n+1}, u_{h}^{n, i}, i=1, \ldots, q$, be as in (2.13) and (2.14), and let $\left\{\tilde{\phi}_{i}\right\}_{i=1}^{N}$ be the basis introduced in $\S 2$. Then there exist unique $N$-vectors $U_{h}^{n}$, $U_{h}^{n+1}, U_{h}^{n, i}, i=1, \ldots, q$, such that

$$
\begin{gathered}
u_{h}^{n}=\sum_{j=1}^{N}\left(U_{h}^{n}\right)_{j} \tilde{\phi}_{j}, \quad u_{h}^{n+1}=\sum_{j=1}^{N}\left(U_{h}^{n+1}\right)_{j} \tilde{\phi}_{j}, \\
u_{h}^{n, i}=\sum_{j=1}^{N}\left(U_{h}^{n, i}\right)_{j} \tilde{\phi}_{j}, \quad i=1, \ldots, q .
\end{gathered}
$$

We now have

Theorem 4.2. Suppose that the IRK method satisfies $(\mathrm{S})$ and $(\mathrm{P})$. Then there exists a constant $c$ independent of $k$ and $h$ such that

$$
\begin{aligned}
& \left(\tilde{G}\left[U^{n+1}-U_{h}^{n+1}\right], U^{n+1}-U_{h}^{n+1}\right)_{h} \\
& \quad \leq(1+c k)\left(\widetilde{G}\left[U^{n}-U_{h}^{n}\right], U^{n}-U_{h}^{n}\right)_{h}+c k h^{2 r} .
\end{aligned}
$$

Proof. From (3.2) we get

$$
\widetilde{G} U^{n+1}=\widetilde{G} U^{n}-k \sum_{l=1}^{q} b_{l} \widetilde{G}\left(U^{n, l} U_{x}^{n, l}+U_{x x x}^{n, l}\right) .
$$

Define the map $f: S_{h}^{r} \times S_{h}^{r} \rightarrow S_{h}^{r}$ by $(f(v, w), \chi)=h^{-1}\left(v w_{x}+w_{x x x}, \chi\right)$, $\forall \chi \in S_{h}^{r}$. Such a map exists by virtue of the Riesz representation theorem. Denote $f(v, v)$ by $f(v)$. The map $f(\cdot)$ induces a unique map $\widetilde{F}: R^{N} \rightarrow R^{N}$ via

$$
(\widetilde{F}(V))_{i}=\left(f(v), \tilde{\phi}_{i}\right)=h^{-1}\left(v v_{x}+v_{x x x}, \tilde{\phi}_{i}\right), \quad i=1, \ldots, N,
$$


where $v=\sum_{j=1}^{N} V_{j} \tilde{\phi}_{j}$. Now from (2.13),

$$
\widetilde{G} U_{h}^{n+1}=\widetilde{G} U_{h}^{n}-k \sum_{i=1}^{q} b_{i} \widetilde{F}\left(U_{h}^{n, i}\right) .
$$

From (4.23) and (4.21) we get

$$
\widetilde{G} E^{n+1}=\widetilde{G} E^{n}-k \sum_{i=1}^{q} b_{i}\left[\Xi^{n, i}-Z^{n, i}\right]
$$

with $E^{n+1}=U^{n+1}-U_{h}^{n+1}, E^{n}=U^{n}-U_{h}^{n}, \Xi^{n, i}=\widetilde{F}\left(U^{n, i}\right)-\widetilde{F}\left(U_{h}^{n, i}\right)$, and $Z^{n, i}=\widetilde{F}\left(U^{n, i}\right)-\widetilde{G}\left[U^{n, i} U_{x}^{n, i}+U_{x x x}^{n, i}\right]$. Note that $\left(\widetilde{F}\left(U^{n, i}\right)\right)_{j}=\left(f\left(\tilde{u}_{h}^{n, i}\right), \tilde{\phi}_{j}\right)$, $j=1, \ldots, N$, where $\tilde{u}_{h}^{n, i}$ is the quasi-interpolant of $u^{n, i}$ given by $\tilde{u}_{h}^{n, i}=$ $\sum_{j=1}^{N} U_{j}^{n, i} \tilde{\phi}_{j}$. From (4.24) we get

$$
\begin{aligned}
\left(\widetilde{G} E^{n+1}\right. & \left., E^{n+1}\right)_{h} \\
= & \left(\widetilde{G} E^{n}, E^{n}\right)_{h}-2 k \sum_{i=1}^{q} b_{i}\left(\widetilde{G}^{1 / 2} E^{n}, \widetilde{G}^{-1 / 2}\left[\Xi^{n, i}-Z^{n, i}\right]\right)_{h} \\
& +k^{2} \sum_{i, l=1}^{q} b_{i} b_{l}\left(\widetilde{G}^{-1 / 2}\left[\Xi^{n, i}-Z^{n, i}\right], \widetilde{G}^{-1 / 2}\left[\Xi^{n, l}-Z^{n, l}\right]\right)_{h} .
\end{aligned}
$$

Now from (3.1) we get

$$
\widetilde{G} U^{n, i}=\widetilde{G} U^{n}-k \sum_{j=1}^{q} a_{i j} \widetilde{G}\left(U^{n, j} U_{x}^{n, j}+U_{x x x}^{n, j}\right), \quad i=1, \ldots, q .
$$

From (2.14) we also get

$$
\widetilde{G} U_{h}^{n, i}=\tilde{G} U_{h}^{n}-k \sum_{j=1}^{q} a_{i j} \tilde{F}\left(U_{h}^{n, j}\right) .
$$

From (4.26) and (4.27) we get, with $E^{n, i}=U^{n, i}-U_{h}^{n, i}$,

$$
\widetilde{G}^{1 / 2} E^{n, i}=\widetilde{G}^{1 / 2} E^{n}-k \sum_{j=1}^{q} a_{i j} \widetilde{G}^{-1 / 2}\left[\Xi^{n, j}-Z^{n, j}\right] .
$$

Using (4.28) in (4.25), we get

$$
\begin{aligned}
\left(\widetilde{G} E^{n+1}, E^{n+1}\right)_{h}= & \left(\widetilde{G} E^{n}, E^{n}\right)_{h}-2 k \sum_{i=1}^{q} b_{i}\left(\widetilde{G}^{1 / 2} E^{n, i}, \widetilde{G}^{-1 / 2}\left[\Xi^{n, i}-Z^{n, i}\right]\right)_{h} \\
& -k^{2} \sum_{i, j=1}^{q} m_{i j}\left(\widetilde{G}^{-1 / 2}\left[\Xi^{n, i}-Z^{n, i}\right], \tilde{G}^{-1 / 2}\left[\Xi^{n, j}-Z^{n, j}\right]\right)_{h} .
\end{aligned}
$$

Since the matrix $\left\{m_{i j}\right\}$ is nonnegative definite, we obtain

$$
\left(\widetilde{G} E^{n+1}, E^{n+1}\right)_{h} \leq\left(\widetilde{G} E^{n}, E^{n}\right)_{h}-2 k \sum_{i=1}^{q} b_{i}\left(E^{n, i}, \Xi^{n, i}-Z^{n, i}\right)_{h}
$$


We next estimate the term $\left(E^{n, i}, \Xi^{n, i}\right)_{h}$. Note that

$$
\begin{aligned}
\left(E^{n, i}, \Xi^{n, i}\right)_{h} & =h \sum_{j=1}^{N}\left(U^{n, i}-U_{h}^{n, i}\right)_{j}\left(\widetilde{F}\left(U^{n, i}\right)-\widetilde{F}\left(U_{h}^{n, i}\right)\right)_{j} \\
& =\left(\tilde{u}_{h}^{n, i} \tilde{u}_{h x}^{n, i}+\tilde{u}_{h x x x}^{n, i}-\left[u_{h}^{n, i} u_{h x}^{n, i}+u_{h x x x}^{n, i}\right], \tilde{u}_{h}^{n, i}-u_{h}^{n, i}\right) \\
& =\frac{1}{2}\left(\tilde{u}_{h x}^{n, i},\left[\tilde{u}_{h}^{n, i}-u_{h}^{n, i}\right]^{2}\right) \leq \frac{1}{2}\left\|\tilde{u}_{h x}^{n, i}\right\|_{\infty}\left\|\tilde{u}_{h}^{n, i}-u_{h}^{n, i}\right\|^{2}
\end{aligned}
$$

Now note that

$$
\left\|\tilde{u}_{h}^{n, i}-u_{h}^{n, i}\right\|^{2}=\left(\tilde{G}\left[U^{n, i}-U_{h}^{n, i}\right], U^{n, i}-U_{h}^{n, i}\right)_{h}=\left(\widetilde{G} E^{n, i}, E^{n, i}\right)_{h} .
$$

Also, using (2.6), (3.3), (3.4), and (3.25), we have

$$
\left\|\tilde{u}_{h x}^{n, i}\right\|_{\infty} \leq c\left(\left\|u^{0}\right\|_{r}\right) .
$$

Hence,

$$
\left(E^{n, i}, \Xi^{n, i}\right)_{h} \leq c\left(\widetilde{G} E^{n, i}, E^{n, i}\right)_{h}
$$

Now for $j=1, \ldots, N$

$$
\begin{aligned}
& \left(Z^{n, i}\right)_{j}=\left(\widetilde{F}\left(U^{n, i}\right)-\widetilde{G}\left[U^{n, i} U_{x}^{n, i}+U_{x x x}^{n, i}\right]\right)_{j} \\
& =h^{-1}\left(\tilde{u}_{h}^{n, i} \tilde{u}_{h x}^{n, i}+\tilde{u}_{h x x x}^{n, i}, \tilde{\phi}_{j}\right)-h^{-1} \sum_{l=1}^{N}\left(\tilde{\phi}_{l}, \tilde{\phi}_{j}\right)\left(u^{n, i} u_{x}^{n, i}+u_{x x x}^{n, i}\right)(l h) \\
& =h^{-1}\left\{\left(\left[\tilde{u}_{h}^{n, i}-u^{n, i}\right] \tilde{u}_{h x}^{n, i}, \tilde{\phi}_{j}\right)+\left(u^{n, i} \tilde{u}_{h x}^{n, i}+\tilde{u}_{h x x x}^{n, i}, \tilde{\phi}_{j}\right)\right. \\
& \left.-\sum_{l=1}^{N}\left(\tilde{\phi}_{l}, \tilde{\phi}_{j}\right)\left(u^{n, i} u_{x}^{n, i}+u_{x x x}^{n, i}\right)(l h)\right\} \\
& =h^{-1}\left\{\left(\left[\tilde{u}_{h}^{n, i}-u^{n, i}\right] \tilde{u}_{h x}^{n, i}, \tilde{\phi}_{j}\right)-\sum_{l=1}^{N}\left[\left(\tilde{\phi}_{l}, \tilde{\phi}_{j}\right)\left(P\left(x, u^{n, i}, D\right) u^{n, i}\right)(l h)\right.\right. \\
& \left.\left.-B\left(\tilde{\phi}_{l}, \tilde{\phi}_{j}\right) u^{n, i}(\ln )\right]\right\}
\end{aligned}
$$

where $P\left(x, u^{n, i}, D\right)$ represents the differential operator $u^{n, i} \partial / \partial x+\partial^{3} / \partial x^{3}$ and $B\left(\tilde{\phi}_{l}, \tilde{\phi}_{j}\right)=\left(P\left(x, u^{n, i}, D\right) \tilde{\phi}_{l}, \tilde{\phi}_{j}\right)$. The term

$$
h^{-1} \sum_{l=1}^{N}\left(\tilde{\phi}_{l}, \tilde{\phi}_{j}\right)\left(P\left(x, u^{n, i}, D\right) u^{n, i}\right)(l h)-B\left(\tilde{\phi}_{l}, \tilde{\phi}_{j}\right) u^{n, i}(l h)
$$

is precisely the truncation error $F_{j}$ introduced by Thomée and Wendroff (cf. [12, p. 1064]). By Lemma 4.2 of [12],

$$
\max _{1 \leq j \leq N}\left|F_{j}\right| \leq c h^{r}
$$


Hence,

$$
\begin{aligned}
\left(E^{n, i}, Z^{n, i}\right)_{h} & =h \sum_{j=1}^{N}\left(U^{n, i}-U_{h}^{n, i}\right)_{j}\left(Z^{n, i}\right)_{j} \\
& =\left(\tilde{u}_{h x}^{n, i}\left[\tilde{u}_{h}^{n, i}-u^{n, i}\right], \tilde{u}_{h}^{n, i}-u_{h}^{n, i}\right)-h \sum_{j=1}^{N} F_{j}\left(E^{n, i}\right)_{j} \\
& \leq\left\|\tilde{u}_{h x}^{n, i}\right\|_{\infty}\left\|\tilde{u}_{h}^{n, i}-u^{n, i}\right\|\left\|\tilde{u}_{h}^{n, i}-u_{h}^{n, i}\right\|+\|F\|_{h}\left\|E^{n, i}\right\|_{h} .
\end{aligned}
$$

Using (4.33), (2.6), (2.5), and (2.3) in (4.34), we get

$$
\left(E^{n, i}, Z^{n, i}\right)_{h} \leq c h^{2 r}+c\left(\widetilde{G} E^{n, i}, E^{n, i}\right)_{h} .
$$

Using (4.35) and (4.31) in (4.29), we get

$$
\left(\widetilde{G} E^{n+1}, E^{n+1}\right)_{h} \leq\left(\widetilde{G} E^{n}, E^{n}\right)_{h}+c k \sum_{i=1}^{q}\left(\widetilde{G} E^{n, i}, E^{n, i}\right)_{h}+c k h^{2 r} .
$$

Since the matrix $D A^{-1} D^{-1}$ is positive definite, applying a previously used diagonalization technique to (4.28) yields via (4.31) and (4.35)

$$
\sum_{i=1}^{q}\left(\widetilde{G} E^{n, i}, E^{n, i}\right)_{h} \leq c\left(\widetilde{G} E^{n}, E^{n}\right)_{h}+c k h^{2 r} \text {. }
$$

Using this in (4.36) gives (4.20).

The main result of this paper now follows.

Theorem 4.3. Suppose $u_{h}^{0} \in S_{h}^{r}$ satisfies (2.12). Under the hypotheses of Theorems 4.1 and 4.2, we have

$$
\max _{0 \leq n \leq J}\left\|u\left(t^{n}\right)-u_{h}^{n}\right\| \leq c\left(k^{\nu}+h^{r}\right)
$$

for some constant $c$ independent of $k$ and $h$.

Proof. Let $U\left(t^{n+1}\right)$ denote the $N$-vector with components $u\left(j h, t^{n+1}\right), j=$ $1, \ldots, N$. Recall that $U^{n}=U\left(t^{n}\right)$ here. From (4.20), (4.9), and (2.3),

$$
\begin{aligned}
&\left(\tilde{G}\left[U\left(t^{n+1}\right)-U_{h}^{n+1}\right], U\left(t^{n+1}\right)-U_{h}^{n+1}\right)_{h} \\
&=\left(\widetilde{G}\left[U^{n+1}-U_{h}^{n+1}\right], U^{n+1}-U_{h}^{n+1}\right)_{h} \\
& \quad+\left(\widetilde{G}\left[U\left(t^{n+1}\right)-U^{n+1}\right], U\left(t^{n+1}\right)-U^{n+1}\right)_{h} \\
& \quad+2\left(\widetilde{G}\left[U\left(t^{n+1}\right)-U^{n+1}\right], U^{n+1}-U_{h}^{n+1}\right)_{h} \\
& \leq(1+k)\left(\widetilde{G}\left[U^{n+1}-U_{h}^{n+1}\right], U^{n+1}-U_{h}^{n+1}\right)_{h} \\
&+\left(1+k^{-1}\right)\left(\widetilde{G}\left[U\left(t^{n+1}\right)-U^{n+1}\right], U\left(t^{n+1}\right)-U^{n+1}\right)_{h} \\
& \leq(1+c k)\left(\widetilde{G}\left[U\left(t^{n}\right)-U_{h}^{n}\right], U\left(t^{n}\right)-U_{h}^{n}\right)_{h}+c k h^{2 r}+c k^{2 \nu+1} .
\end{aligned}
$$

From this it follows that

$$
\begin{aligned}
& \left(\tilde{G}\left[U\left(t^{n}\right)-U_{h}^{n}\right], U\left(t^{n}\right)-U_{h}^{n}\right)_{h} \\
& \quad \leq c\left(\widetilde{G}\left[U(0)-U_{h}^{0}\right], U(0)-U_{h}^{0}\right)_{h}+c h^{2 r}+c k^{2 \nu}
\end{aligned}
$$


or

$$
\left\|\tilde{u}_{h}\left(t^{n}\right)-u_{h}^{n}\right\| \leq c\left\|\tilde{u}_{h}(0)-\pi_{h} u^{0}\right\|+c\left(h^{r}+k^{\nu}\right),
$$

where $\tilde{u}_{h}(t)$ is the quasi-interpolant of $u(t)$. (4.37) now follows from (4.38), (2.5), and (2.12).

\section{BIBLIOGRAPHY}

1. R. A. Adams, Sobolev spaces, Academic Press, New York, 1970.

2. G. A. Baker, V. A. Dougalis, and O. A. Karakashian, Convergence of Galerkin approximations for the Korteweg-de Vries equation, Math. Comp. 40 (1983), 419-433.

3. J. L. Bona, J. A. Dougalis, and O. A. Karakashian, Conservative high order schemes for the generalized Korteweg-de Vries equation (submitted).

4. J. L. Bona and R. Smith, The initial-value problem for the Korteweg-de Vries equation, Philos. Trans. Roy. Soc. London Ser. A 278 (1975), 555-604.

5. J. C. Butcher, Implicit Runge-Kutta processes, Math. Comp. 18 (1964), 60-64.

6. M. Crouzeix, Sur l'approximation des équations différentielles opérationnelles linéaires par des méthodes de Runge-Kutta, Thèse, Université Paris VI, 1975.

7. K. Dekker and J. G. Verwer, Stability of Runge-Kutta methods for stiff nonlinear differential equations, CWI Monographs, North-Holland, Amsterdam, 1984.

8. V. A. Dougalis and O. A. Karakashian, On some high-order accurate fully discrete Galerkin methods for the Korteweg-de Vries equation, Math. Comp. 45 (1985), 329-345.

9. P. Hartman, Ordinary differential equations, Wiley, New York, 1964.

10. O. A. Karakashian, On Runge-Kutta methods for parabolic problems with time-dependent coefficients, Math. Comp. 47 (1986), 77-101.

11. O. A. Karakashian and W. Rust, On the parallel implementation of implicit Runge-Kutta methods, SIAM J. Sci. Statist. Comput. 9 (1988), 1085-1090.

12. V. Thomée and B. Wendroff, Convergence estimates for Galerkin methods for variable coefficient initial value problems, SIAM J. Numer. Anal. 11 (1974), 1059-1068.

Department of Mathematics, University of Tennessee, Knoxville, Tennessee 379961300

E-mail address: ohannes@sun2.math.utk.edu 